\title{
Operation Mode and Switching Control Design for a Solar Energy Fresh Air System
}

\author{
Lili Wang ${ }^{1,2} a^{*}$, Naixiu Ding ${ }^{1, b}$ and Meijia Wang ${ }^{3, c}$ \\ ${ }^{1}$ Qingdao University of Science and Technology, Qingdao, China \\ ${ }^{2}$ Qingdao Hanhe Cable Co., Ltd. Qingdao, China \\ ${ }^{3}$ Qingdao Publishing \& Media Co., Ltd., Qingdao, China \\ awllqust@126.com, bnxding1717@163.com, cw.mj@163.com
}

Keywords: Solar Energy; Fresh Air; Operation Mode; Switching Control.

\begin{abstract}
Concerning with the environmental pollution and energy crisis, a solar energy fresh air heating system coupled with the Latent Heat Thermal Storage (LHTS) tank was developed, in which solar energy was used as heat supply source, and the heat storage material was also employed as the solar energy has intermittent and instability characteristics. In this paper, the operating principle of the solar energy fresh air system was firstly analyzed and the overall control objectives was then proposed; secondly, three operation mode was determined from the point of view of simulating the solar operating conditions. In order to make effective use of solar energy maximizing the utilization ratio, the mode switching control scheme was finally designed according to the mode conversion evidence.
\end{abstract}

\section{Introduction}

The solar energy fresh air system is composed of three parts: solar energy collectors, Latent Heat Thermal Storage tank and fresh air heating surface exchanger. It utilizes the heat exchanger principle during the solar hot water system, the phase change thermal storage system and air/water fresh air system, which effectively realized organized winter room ventilation and solar heating fresh air, so that fresh air has the capacity of part of indoor heating load. It makes the renewable energy apply directly to energy-saving heating and air conditioning system.

\section{Principle and Control Objective Analysis}

System Operation Principle. The sun's radiation heat is collected by the solar collectors to heat the water in pipes, and the heated water flows through the pipes according to the system settings of the operation modes which are controlled by the pumps and valves. The heat could be stored into the phase change thermal storage device or makes heat transfer to fresh air with the fan coil, so that it can realize the functions of fresh air heating, heat storage and effective energy saving of indoor auxiliary heating[1-3].The overall operation principle of solar energy fresh air system is shown in Fig. 1. 


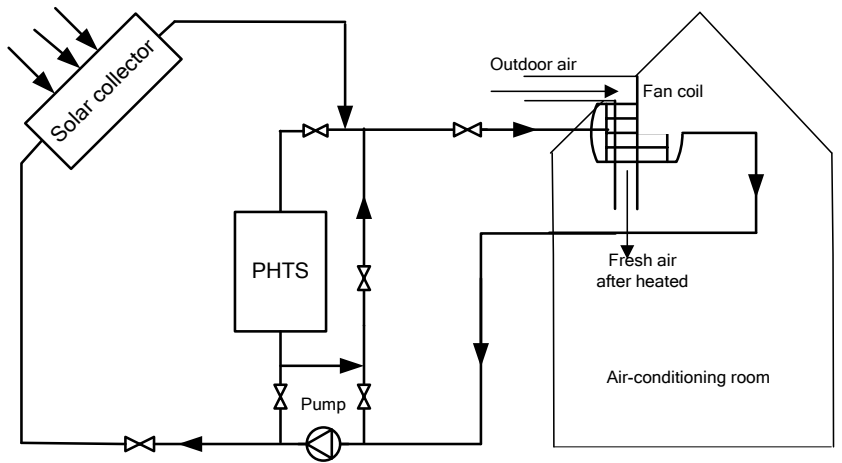

Figure 1. Shematic diagram of the solar fresh air system

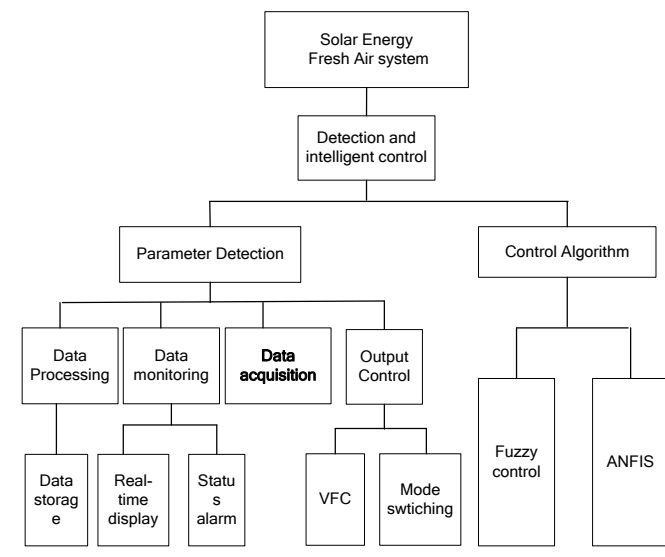

Figure 2. Control process diagram

System Control Objective Analysis. As shown in Fig .2, we have to complete three objectives:

1. The establishment of fresh air automatic control system based on CAN bus which can make detection and control of various physical quantities such as temperature, flow rate, and pressure and so on. These works has been completed [4].

2. The outdoor air (whose temperature is represented by $\mathrm{T}_{\mathrm{ao}}$ ) through the fan coil makes heat transfer with the heated water in pipe. The voltage of the inverter is controlled to adjust pump speed, so as to control the fan coil heat medium water flow into fan coil. Thus the fresh air is heated to set point Ts to be sent into the room. The air temperature control is realized through the intelligent algorithm, which is described in the other paper.

3. The solar energy fresh air system is composed of the heat collecting part, heat storage and releasing part, fresh air part. While the system is on operation, it should detect the environment and its own parameters change in real time so as to automatically switching the operation mode. And the mode switching is the comprehensive effect of different parts of the control system. In this paper, we will discuss the various parts on-off control and mode switching control [5].

\section{Operation Mode Design of Solar Energy Fresh Air System}

For simplification, we determine to simulate the overall solar day operation for three modes according to solar radiation, time-changing heat amount from the collectors: all solar heat for fresh air mode, one part of solar heat for fresh air the other for LHTS mode, all LHTS heat for fresh air mode

All Solar Heat for Fresh Air Mode. As shown in Fig. 3 (The red line represents the water entry while the blue lines represent the backwater), the applicable conditions of mode is that: in the morning or sunlight intensity is not high enough in the daytime, all of the solar heat is used for heating the fresh air to meet the temperature requirements of indoor fresh air
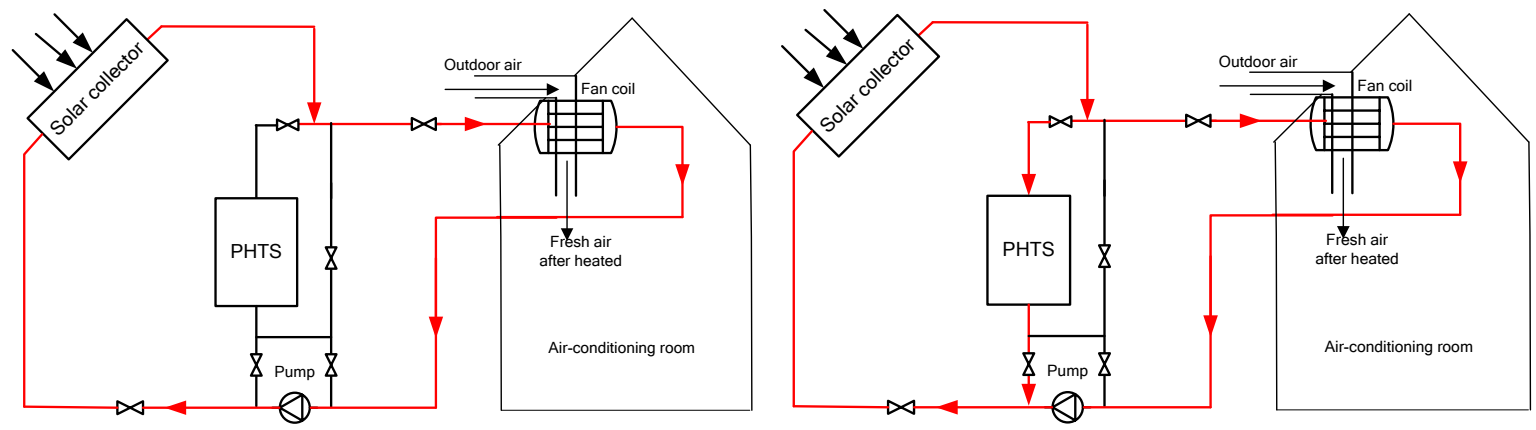

Figure 3. All solar heat for fresh air mode

Figure 4. Part of solar heat for fresh air the other for LHTS mode 
Part of Solar Heat for Fresh Air the other for LHTS Mode. As the intensity of solar irradiation increases during daytime, the heat can not only provide directly to the fan coil for comfortable room air; but the remaining heat can also be stored in the LHTS device, which is shown in Fig. 4.

All LHTS Heat for Fresh Air Mode: All the heat which is stored in LHTS device makes transfer with fan coil. The applicable conditions of mode is that if the solar energy shortage, which is unable to meet the indoor fresh air heating conditions (such as night), all the LHTS heat is used to heat the fresh air (as shown in Fig. 5)

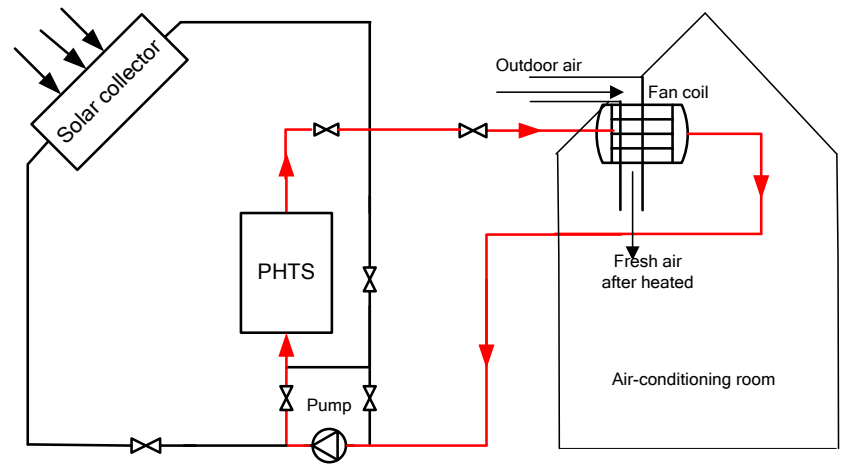

Figure 5. All LHTS heat for fresh air mode

\section{Function and Control of Solar Energy Fresh Air System}

Fan Coil Speed Control. The fan coil connects the fresh air system with air supply room. To achieve the most energy-saving effect, at the same time to ensure the comfort of the room, it is necessary to control the speed of fan coil effectively. In standard VAV system, fresh air for a percentage of the total volume can be maintained, the actual quantity of fresh air will change with the total amount of air. However, in many cases, it should have the minimum fresh air volume [6]. Therefore, the fan coil speed is divided into three gears in this paper: large, medium and small which can be adjusted in accordance with the fresh air volume requirements.

Solar Collector On-off Control. The solar collector is an extremely important part of the fresh air system, which provides heat for the fresh air. In consideration of the heat capacity and the heat loss factor, the system controls the on-off state in the following way:

$$
\begin{aligned}
& \left\{\begin{array}{l}
T_{P} \geq T_{s i} \\
T_{s o}-T_{s i} \geq \Delta T_{s c}
\end{array} \quad \text { OFF } \rightarrow\right. \text { ON } \\
& T_{s o}-T_{s i}<\Delta T_{s c} \quad \mathrm{ON} \rightarrow \mathrm{OFF}
\end{aligned}
$$

in which $T_{P}$ is the solar panel temperature, $T_{s i}$ is the solar collector inlet temperature, $T_{s o}$ is the solar collector outlet temperature, $\Delta T_{s c}$ is the control parameter of solar collector temperature differential.

LHTS Device On-off Control. LHTS device is solar heat storage component of which the regenerative performance also affects the performance of the system. Its operation is divided into two conditions: one is LHTS heat storage while supplying heat for fresh air; the other is LHTS device heat release. In condition 1, the temperature differential between inlet and outlet of the LHTS device (which is represented by temperature differential of the water) is chosen as the control parameter; In condition 2, the outlet temperature of the LHTS device (which is represented by outlet of the water) is used as the parameter. The LHTS device control method is specifically described as follows: 
Heat storage $\begin{cases}\Delta T_{f} \geq \Delta T_{f c} & \text { ON } \\ \Delta T_{f}<\Delta T_{f c} & \text { OFF }\end{cases}$

Heat discharge $\begin{cases}T_{f o} \geq T_{f c} & \text { ON } \\ T_{f o}<T_{f c} & \text { OFF }\end{cases}$

\section{Operation Mode Switching Control of Solar Energy Fresh Air System}

Mode Switching Evidence. The system operation mode conversion mainly depends on the properties of fan coil and LHTS device besides on-off control of various components described in previous section.

Static Heat Transfer Equation. For there is no phase change between two fluid (air, water) through the fan coil, heat that the hot water gives out should be equal to the heat absorbed by the fresh air without considering the heat loss.

$$
G_{f} \mathrm{c}_{f}\left(T_{f i}-T_{f 0}\right)=G_{a} \mathrm{c}_{a}\left(T_{a \mathrm{o}}-T_{a \mathrm{i}}\right)
$$

In which $G_{f}$, the heated water flowrate; $c_{f}$, the average specific heat capacity within the range of inlet and outlet temperature; $T_{f i}$, the inlet temperature of fan coil; $T_{f_{0}}$, the outlet temperature of fan coil; $G_{a}$, the fresh air flow; $\mathrm{c}_{a}$, the air specific heat; $T_{a \mathrm{i}}$, the fresh air inlet temperature; $T_{a 0}$ the fresh air outlet temperature.

Operation Mode Conversion Control. The operation condition of each mode of the system is decided by the on-off state of each part of the system, and each operating mode is the combination of various compoents of the system running state. Simulating the solar energy fresh air system in a day, the transition of operation mode is shown in Fig. 6.

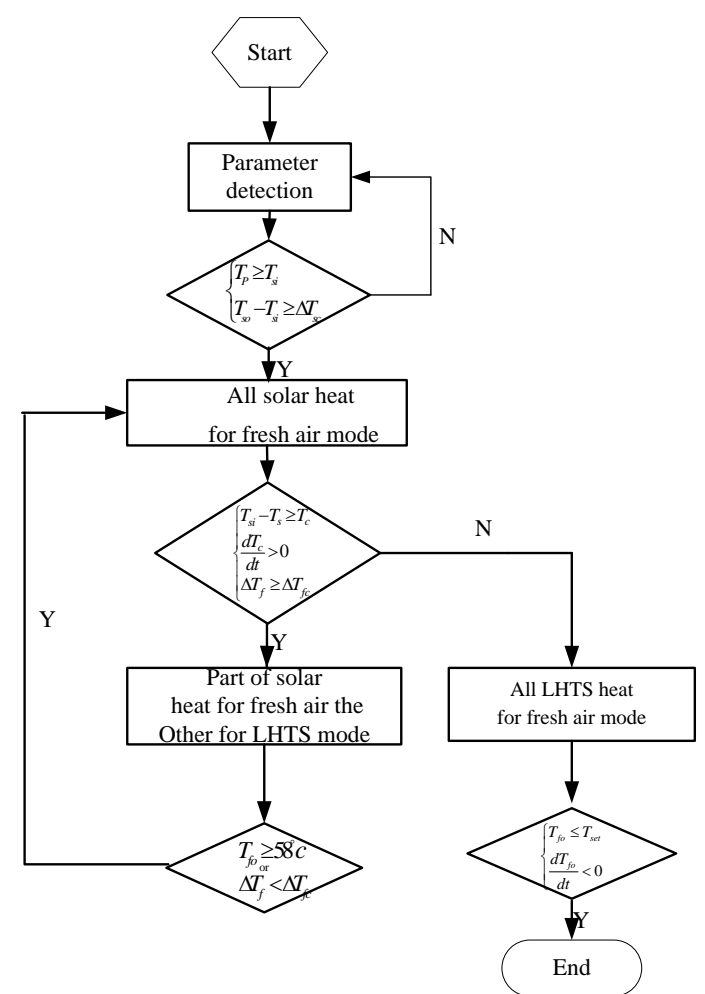

Figure 6. Mode switching diagram for solar energy fresh air system 
Mode1 to Mode2. With the sun light intensity more and more high, the minimum flow of pipe heat water provides heat greater than or equal to the fresh air heat demand, i.e.:

$$
G_{f m i n} \mathrm{c}_{f}\left(T_{f \mathrm{i}}-T_{f \mathrm{o}}\right) \geq G_{a} \mathrm{c}_{a}\left(T_{a \mathrm{i}}-T_{a \mathrm{o}}\right)
$$

$$
\left\{\begin{array}{l}
T_{f i}-T_{s e t} \geq T_{c 1} \\
\frac{d T_{c 1}}{d t}>0
\end{array}\right.
$$

Mode2 to Mode1. In mode2, if the temperature differential between the outlet and inlet is smaller than the temperature control parameters, and if the outlet temperature is larger than $58^{\circ} \mathrm{C}$ [1], it is said that the heat storage is accomplished, and the device stops storing heat. The whole system is converted to mode1.

$$
\left\{\begin{array}{l}
\mathrm{T}_{f o} \geq 58^{\circ} \mathrm{c} \\
\Delta T_{f}<\Delta T_{f c}
\end{array}\right.
$$

Mode1 to Mode3. In mode1, if the detected fan coil water inlet temperature $\left(T_{f i}\right)$ is lower than fresh air setpoint $\left(T_{s e t}\right) 5^{\circ} \mathrm{C}\left(T_{c 2}\right)$, and has a downward trend, indicating that the heat of the sun light is not enough to provide for fresh air, the operation mode 1 should switch to mode3.

$$
\left\{\begin{array}{l}
T_{f i}-T_{s e t}<T_{c 2} \\
\frac{d T_{c 2}}{d t}<0
\end{array}\right.
$$

If the water temperature of outlet heat storage device is smaller than or equal to the fresh air set point, and has the downward trend, it represents the end of the exothermic heat storage device, and overall operation modes of fresh air system simulating a solar day is completed.

$$
\left\{\begin{array}{l}
T_{f o} \leq T_{s e t} \\
\frac{d T_{f o}}{d t}<0
\end{array}\right.
$$

\section{Summary}

In this paper, we focus on a solar energy fresh air system as a new kind of energy saving strategy. Firstly, we analyzed the system working principle and structure, and put forward three modes and its operating conditions. Finally, from the point of view of maximizing the solar thermal utilization rate, we designed the strategy of conversion between three kinds of modes.

\section{Acknowledgements}

In this paper, the research was supported by the Natural Science Foundation of Shandong Province, China (ZR2015PF008)

\section{References}

[1] LI Zhiyong, Theoretical and experimental study on solar heating system with phase change thermal storage [D].Beijing: Beijing University of Technology, 2011.(In Chinese) 
[2] Weber, R; Dorer, V. Long-term heat storage with NaOH. Vacuum, v 82, n 7-14 March 2008, 708-716

[3] Vikram.D, Kaushik. S, Prashanth. V, Nallusamy.N. An improvement in the solar water heating systems by thermal storage using phase change materials. International Solar Energy Conference, Proceedings of the ASME International Solar Energy Conference, ISEC2006, 2007, 409-416.

[4] Zhou, ligong .Application and Practice of iCAN Field Bus. [M].Beijing: Guangzhou Zhiyuan Electronics CO., LTD, 2007.Joerg Christian Wolf, Phil Hall, Paul Robinson, Phil Culverhouse. Bioloid based Humanoid Soccer Robot Design, 2007. (In Chinese)

[5] Wang lili, control system study on solar heating system with phase change thermal storage [D].Beijing: Beijing university of posts and communications,2010. (In Chinese)

[6] Cai jinglang .Variable air volume air conditioning design [M]. Beijing:China Architecture \& Building Press, 2007 